\title{
Spontaneous Formation of Crystalline Lithium Molybdate \\ from Solid Reagents at Room Temperature
}

Thomas W. S. Yip, ${ }^{1}$ Edmund J. Cussen ${ }^{1 *}$ and Claire Wilson ${ }^{2}$

${ }^{1}$ WestCHEM, Department of Pure and Applied Chemistry, Thomas Graham Building, The University of Strathclyde, 295 Cathedral Street, Glasgow, G1 1XL Scotland

${ }^{2}$ Rigaku Europe, Unit B6, Chaucer Business Park, Watery Lane, Kemsing, Sevenoaks, Kent, TN15 6QY UK

E-mail: Edmund.Cussen@strath.ac.uk

Tel: +44 1415482797

Fax: +441415484822 


\begin{abstract}
Lithium molybdate has been prepared by grinding $\mathrm{LiOH} \cdot \mathrm{H}_{2} \mathrm{O}$ with $\mathrm{MoO}_{3}$ in air at room temperature. X-ray powder diffraction data show that the formation of highly crystalline $\mathrm{Li}_{2} \mathrm{MoO}_{4}$ is largely complete after 10 minutes. The phenacite structure of this material is the same as that derived from an X-ray diffraction study of a single crystal obtained from aqueous solution $[R \overline{3} ; \mathrm{a}=14.3178(14) \AA, \mathrm{c}=9.5757(9) \AA]$. Anhydrous lithium hydroxide fails to give the same reaction indicating that the water of crystallisation of $\mathrm{LiOH} \cdot \mathrm{H}_{2} \mathrm{O}$ is a vital component in this rapid synthesis. Differential scanning calorimetry measurements show that this reaction can proceed spontaneously between the two stable solid reagents at sub-ambient temperatures and is driven by the liberation of water from the crystalline lattice. Lithium molybdate prepared in this manner has significantly smaller and more regularly shaped particles than samples prepared by other synthetic methods.
\end{abstract}




\section{Introduction}

Complex metal oxides are typically prepared by heating combinations of binary metal salts in quantities of the appropriate stoichiometry in order to use the entropically-driven mixing of cations to drive the formation of the desired ternary compound. ${ }^{1}$ This high temperature route provides sufficient energy to overcome two of the challenges of reactions between solids: it simultaneously breaks the large number of bonds in the crystalline reagents and it provides the activation energy for ion migration. The latter permits mass transport to be effective over the course of days, rather than the geological periods anticipated at more modest temperatures. Unfortunately, this approach has two significant failings: the use of high temperatures means that usually only the thermodynamically stable product is accessible and that particle growth occurs in an uncontrolled manner during synthesis. In addition to these chemical considerations it should be noted that these reactions often involve temperatures in excess of $1000{ }^{\circ} \mathrm{C}$ for several days at a time and so the energetic costs of this approach to bond breaking and diffusion are considerable. The use of high temperatures can also introduce additional complications where reagents show significant volatility at high temperatures. This can be particularly problematic in the preparation of lithium-containing compounds. $^{2}$

The search for materials that are either kinetically stable, or only thermodynamically stable at lower temperatures, has led to the development of a number of 'soft' synthetic methods; the so-called chemie douche approaches. ${ }^{1}$ These often rely on differing rates of ion mobility to exchange or remove a subset of the ions in the material. Important examples exist in a wide range of compounds including layered intercalation hosts $^{3-6}$ fast ion conductors ${ }^{7,8}$ and electronically-active materials. ${ }^{9-12}$ These routes typically involve preparing a precursor compound at high temperature and then modifying this material; by treatment with a salt 
solution $^{3}$ or electrochemically ${ }^{4}$ to manipulate the cations or with a reducing $9,10,13$ or oxidising agent $^{14}$ to adjust the occupancy of the anion sublattice.

Control of particle morphology often also requires multi-step synthetic processes, for example sol-gel routes ${ }^{15}$ or precipitation, ${ }^{16-18}$ and additional separation and washing stages. All of these processes are time-consuming and labour intensive and involve considerable inefficiencies and waste in the use of solvents or chelating agents as well as the subsequent heating for several hours or days at temperatures up to $1000{ }^{\circ} \mathrm{C}$.

A number of routes have been explored to reduce the reaction time of solid state reactions. Some of these processes have used the formation of a citrate gel or mechanical milling ${ }^{19}$ to manipulate the starting materials in order to reduce the distances required for mass transport. In some cases, high-energy ball-milling is capable of introducing sufficient mechanical energy to cause the formation of crystalline ternary compounds from binary reagents and no subsequent heating is necessary. ${ }^{20,21}$ An alternative, highly successful approach has used high-energy starting materials to access pathways for strongly exothermic reactions. These self-heating reactions can be driven by either metathesis ${ }^{22-24}$ or combustion. ${ }^{25,26}$ Once initiated these reactions proceed rapidly and can be complete within seconds. ${ }^{27}$

The properties of molybdates are of interest for a number of possible applications including corrosion inhibitors ${ }^{28}$ and hosts matrices for lasers. ${ }^{29}$ Lithium molybdate has been shown to be an effective catalyst for the oxidation of methane ${ }^{30}$ and closely related phases are of potential use in solid state lithium batteries as either electrolytes ${ }^{31}$ or electrodes. ${ }^{32}$ A recent report has shown that it is possible to generate nanostructured molybdates via a multi-step precipitation route followed by a $400{ }^{\circ} \mathrm{C}$ heat treatment. ${ }^{18}$

Here we describe a facile, room temperature reaction between two stable crystalline compounds to yield a highly crystalline sample of a ternary phase. This reaction takes place under ambient conditions to yield highly regular particles of $\mu \mathrm{m}$ dimensions and can occur 
without input of either mechanical or thermal energy. The only waste produce is water and as no purification or separation steps are required this reaction represents a considerably greener approach $^{33}$ to synthesis and morphological control of a ternary oxide. Surprisingly this is an endothermic reaction and is driven by entropic considerations. The conditions necessary for this reaction to occur are sufficiently common to suggest that this approach may be applicable to other reactions and provide an extremely efficient alternative to existing multi-step synthetic routes to highly-crystalline continuous-framework materials. 


\section{Experimental}

This paper reports an unusual method for the synthesis of lithium molybdate. In order to better understand the mechanism for this reaction a number of different synthetic routes to lithium molybdate have been explored. All reagents were used as supplied by Alfa Aesar in purities of in excess of $99 \%$ and all reactions were carried out in air under benchtop conditions.

Stoichiometric quantities of lithium hydroxide monohydrate (1.829 g) and molybdenum trioxide $(3.137 \mathrm{~g})$ were ground under ambient conditions in air for one minute using an agate mortar and pestle. This formed a paste that was allowed to dry at room temperature for $c a .1$ hr, leaving a layer of dry product on the surface of the mortar which was dislodged and ground into a fine powder. This preparation yielded a material that will be referred to as sample A. A second preparative route brought the finely-ground reagents into intimate contact with one another with minimal agitation to afford sample B. Lithium molybdate was also prepared following a high-temperature solid state method previously reported to yield an orthorhombic structure. $^{34}$ This synthesis proceeded by heating a pelleted, stoichiometric mixture of lithium carbonate and molybdenum trioxide at temperatures up to $600{ }^{\circ} \mathrm{C}$ to give sample C.

Crystals suitable for structure determination were grown using lithium molybdate prepared in the same manner as sample A. $2.3 \mathrm{~g}$ of $\mathrm{Li}_{2} \mathrm{MoO}_{4}$ was completely dissolved in $20 \mathrm{ml}$ of freshly boiled water at $\mathrm{ca} .70{ }^{\circ} \mathrm{C}$. This solution was boiled in an open flask for a period for $c a$. ten minutes. At this point small crystals were visible and the solution was removed from the heat and allowed to cool to room temperature.

X-ray diffraction data were collected from a colourless columnar crystal, of dimensions 0.30 $\times 0.10 \times 0.10 \mathrm{~mm}^{3}$, mounted on a glass fibre using oil. X-ray intensity data were measured at 150(2) $\mathrm{K}$ on a three-circle diffractometer equipped with a Bruker SMART APEX CCD area 
detector, a graphite monochromator and a Mo $\mathrm{K}_{\alpha}$ sealed tube $(\lambda=0.71073 \AA)$. The structure was solved by direct methods using SHELXS-97 and refined by least-squares methods on $\mathrm{F}^{2}$ using SHELXL-97. ${ }^{35}$ Grinding a batch of crystals grown in this manner gave a polycrystalline material, sample D.

X-ray powder diffraction data were collected from samples contained in an aluminium sample holder using a Siemens D500 diffractometer operating with $\mathrm{Cu} \mathrm{K}_{\alpha}$ radiation in Bragg Brentano geometry. Data were collected at room temperature over the range $10^{\circ} \leq 2 \theta \leq 80^{\circ}$ using a step size of $\Delta 2 \theta=0.02^{\circ}$. The observed data were analysed by the Rietveld method, ${ }^{36}$ as implemented in the GSAS suite of programs, ${ }^{37}$ using pseudo-Voigt and shifted Chebyshev functions to describe the peak shape and background, respectively.

The temperature stability of sample A was evaluated using a Perkin Elmer TGA 7 thermal gravimetric analyser. Samples contained in platinum pans were equilibrated at $38{ }^{\circ} \mathrm{C}$ and then heated to $50{ }^{\circ} \mathrm{C}$ then held isothermally for 5 mins before being heated to $500{ }^{\circ} \mathrm{C}$ at a rate of $10{ }^{\circ} \mathrm{C} \mathrm{min}{ }^{-1}$. The experiment was conducted under a dynamic helium atmosphere.

Electron micrographs were collected using an FEI Sirion 200 scanning electron microscope from samples loaded onto conductive carbon pads.

Calorimetry measurements were carried out using a TA Instruments Q1000 differential scanning calorimeter under a dynamic atmosphere of air. Lithium hydroxide monohydrate and molybdenum oxide were both finely ground and separately placed in an aluminium pan at room temperature. A card barrier was used to physically separate the reagents whilst they were cooled and reached thermal equilibrium at $-80{ }^{\circ} \mathrm{C}$. Once the reagents had equilibrated at this temperature the barrier between the reagents was removed and the two powders were quickly and gently mixed. During the mixing the temperature of the aluminium pan did not rise above $-60{ }^{\circ} \mathrm{C}$. The mixture was re-equilibrated at $-80{ }^{\circ} \mathrm{C}$ before being heated at a rate of $1{ }^{\circ} \mathrm{C} \min ^{-1}$ to $250{ }^{\circ} \mathrm{C}$, and held at this temperature for 5 minutes. X-ray powder diffraction 
data were subsequently collected from the resulting material. The experiment was repeated with fresh reagents and heated to a maximum temperature of $250{ }^{\circ} \mathrm{C}$ before being cooled to $80{ }^{\circ} \mathrm{C}$ and heated to $250{ }^{\circ} \mathrm{C}$ again in order to check the reversibility of the thermal processes observed in the temperature range $-56 \leq \mathrm{T} /{ }^{\circ} \mathrm{C} \leq 26$. Additional experiments incorporating isothermal periods and/or different ramp rates were also carried out to investigate whether the overlapping events occurring in this temperature range could be separated.

\section{Results}

Previous reports on the structure of lithium molybdate have suggested two possible spacegroup assignments: $\mathrm{R} \overline{3}^{38}$ and $\mathrm{P} 3{ }_{2}{ }^{39}$ As shown in Figure 1 the differences in atomic positions between these two structural models are subtle. A detailed examination of these two models shows that they both describe the same phenacite structure-type. However, the implications of the chiral space group $\mathrm{P} 3_{2}$ are of considerable importance in determining the physical properties of lithium molybdate and so in order to resolve this ambiguity, a redetermination of the crystal structure of lithium molybdate was performed.

The structure was solved using single crystal X-ray diffraction data, 867 unique reflections in total, in the space group $R \overline{3}(\mathrm{a}=14.3178(14) \AA, \mathrm{c}=9.5757(9) \AA)$. The final full matrix least-squares refinement on $\mathrm{F}^{2}$ for 65 variables using 867 reflections, positional and anisotropic displacement parameters refined for all atoms, converged at $R(\mathrm{~F})=0.016$ and $w R\left(\mathrm{~F}^{2}\right)=0.036$ with $\mathrm{S}=1.259$. The largest peak on the final difference map was $0.39 \mathrm{e} \AA^{-3}$ and the largest hole was -0.38 e $\AA^{-3}$. This final structural model provides a good fit to the data and is found to be in agreement with the structure determination by Kolitsch. ${ }^{38}$ The crystallographic details and structural parameters are given in Tables 1 and 2, respectively. The small residual electron density indicates that no guest water molecules are present in the 
channels of the structure. This finding was confirmed by thermogravimetric analyses which showed negligible mass loss on heating to $500{ }^{\circ} \mathrm{C}$.

X-ray powder diffraction data collected from sample A identified the bulk phase was lithium molybdate suggesting that reaction (1) had occurred.

$$
2 \mathrm{LiOH} \cdot \mathrm{H}_{2} \mathrm{O}+\mathrm{MoO}_{3} \rightarrow \mathrm{Li}_{2} \mathrm{MoO}_{4}+3 \mathrm{H}_{2} \mathrm{O}
$$

These data could be indexed using the structural model derived from the X-ray single crystal study of lithium molybdate with two additional weak Bragg peaks arising from the presence of $0.7 \mathrm{wt} \%$ of molybdenum trioxide impurity and a second unidentified phase. The data were fitted using the model indicated by the single crystal diffraction study and a total of 15 parameters were refined. The lattice parameters were allowed to vary and showed a difference from the single crystal study that reflects the thermal expansion of the material on heating the phase from the temperature employed for the single crystal diffraction experiment, $150 \mathrm{~K}$, to room temperature. Attempts to refine the atomic coordinates did not provide a significant improvement to the fit and so the final refinement used the atomic parameters derived from the single crystal X-ray diffraction data to give the fit shown in Figure 2. X-ray diffraction data collected from samples $\mathbf{B}, \mathbf{C}$ and $\mathbf{D}$ could be fitted in the same way. The lattice parameters were freely refined against each of these data sets and showed no significant variation between the samples prepared by different routes.

In order to assess the rapidity of the reaction the reagents were ground for one minute and a series of X-ray diffraction patterns were collected immediately. Several of these data sets are collected in Figure 3. These data show that lithium molybdate composes $\geq 98 \%$ of the sample within ten minutes of the reagents coming into contact. Data collected after 50 minutes showed that lithium molybdate composed $>99 \mathrm{wt} \%$ of the sample and resembled the data collected from sample A. No significant difference was observed in the widths of the Bragg peaks of this material compared to any of the other preparations indicating that this 
reaction produces $\mathrm{Li}_{2} \mathrm{MoO}_{4}$ in a highly crystalline state, rather than by slow aging of an amorphous intermediate.

In order to examine the role of water in the reaction a similar preparation was undertaken using anhydrous lithium hydroxide and molybdenum trioxide. One day after mixing these reagents with extensive grinding, lithium molybdate was present as a minority phase, 12 wt\%. X-ray diffraction data collected five months after mixing the reagents showed that molybdenum trioxide remained the majority phase.

Calorimetric measurements were used to follow the reaction between lithium hydroxide monohydrate and molybdenum trioxide that were mixed in situ at $-80{ }^{\circ} \mathrm{C}$. Figure 4 shows data collected between -80 and $250{ }^{\circ} \mathrm{C}$ and clearly shows the presence of an exotherm followed by an endotherm as the sample is heated through $0{ }^{\circ} \mathrm{C}$ and another endotherm in the range $26 \leq \mathrm{T} /{ }^{\circ} \mathrm{C} \leq 49$. Repeating the heating process with extended isothermal periods after the initiation of the exotherm around $3{ }^{\circ} \mathrm{C}$ showed that the exothermic and endothermic processes could not be separated, i.e. once the exothermic process commences the endothermic event will occur. Cooling the mixture from temperatures above these transitions and reheating the sample showed that both of these processes are irreversible. Lithium molybdate shows no significant features in this temperature range.

Calorimetric measurements on the individual reagents and the lithium molybdate product showed no significant thermal transitions on heating the materials from $-80{ }^{\circ} \mathrm{C}$ to room temperature indicating that the initial exotherm and endotherm arise from the reaction between $\mathrm{LiOH} \cdot \mathrm{H}_{2} \mathrm{O}$ and $\mathrm{MoO}_{3}$.

As the final product of this reaction is anhydrous $\mathrm{Li}_{2} \mathrm{MoO}_{4}$ it follows that the water produced by reaction (1) must be vaporised during heating. This substantial endothermic process must occur in the temperature range $26 \leq \mathrm{T} /{ }^{\circ} \mathrm{C} \leq 49$ as no other features are detected below the boiling point of water. However, X-ray powder diffraction data collected from the product of 
these calorimetry measurements show that some molybdenum trioxide reagent remains indicating that the conversion to lithium molybdate is incomplete. Lithium hydroxide monohydrate shows a substantial endotherm around $45{ }^{\circ} \mathrm{C}$ due to the loss of water of crystallisation and so the incomplete reaction means that the endothermic transition between $26{ }^{\circ} \mathrm{C}$ and $49{ }^{\circ} \mathrm{C}$ will include the decomposition of unreacted lithium hydroxide monohydrate. Consequently it is not possible to quantify the progress of the reaction from these measurements.

Scanning electron micrographs shown in Figure 5 reveal considerable variation in particle morphology between the four different preparations. Samples A and B made by room temperature reaction between solid reagents show a relatively narrow distribution of particle size in the range 0.1 to $8 \mu \mathrm{m}$. Sample A contains a regular distribution of smooth, columnar particles with an aspect ratio of approximately four. Sample B contains particles of a similar shape to those in $\mathbf{A}$, but displays a wider range of particles sizes about a larger mean size. However, both of these preparations afford a much more homogeneous sample than recrystallisation from aqueous solution which gives a large variation in particle size from 0.1 to $80 \mu \mathrm{m}$. Lithium molybdate that has been subjected to heating to $600{ }^{\circ} \mathrm{C}$ in a conventional solid state preparation shows dense, monolithic blocks of fused material of several hundred $\mu \mathrm{m}$ in size.

\section{Discussion}

The crystal structure of $\mathrm{Li}_{2} \mathrm{MoO}_{4}$ is best described in the space group $\mathrm{R} \overline{3}$ as previously reported by Zachariasen ${ }^{40}$ and Kolitsch. ${ }^{38}$ A reported orthorhombic unit cell is incompatible with both the single crystal and powder diffraction data. ${ }^{34}$ It is noteworthy that an alternative description of the structure using a trigonal cell ${ }^{39}$ is capable of indexing the powder diffraction data. Although the rhombohedral and trigonal cell have the same dimensions and 
therefore peak positions, the rhombohedral cell is centred and can equally well be described with a primitive cell with $1 / 3$ of the volume. This centring leads to two thirds of the possible reflections being systematically absent, thus the diffraction patterns differ considerably in the number of allowed Bragg reflections. Detailed examination of the structures described in the $\mathrm{P} 32$ and $\mathrm{R} \overline{3}$ cells show that the differences between these two structures are small; in both cases the structure is built up by the same manner, with $\mathrm{MO}_{4}$ tetrahedra connected by cornersharing via trigonally-coordinated oxide anions to 8 neighbouring tetrahedral units. These oxide tetrahedra are occupied in a fully-ordered manner such that each $\mathrm{MoO}_{4}$ unit has 8 nearest-neighbouring $\mathrm{LiO}_{4}$ units, whilst each $\mathrm{LiO}_{4}$ tetrahedra has four $\mathrm{LiO}_{4}$ and four $\mathrm{MoO}_{4}$ neighbours. The small differences between the $\mathrm{P} 3_{2}$ and $\mathrm{R} \overline{3}$ structures arise from variable displacements in the oxide anion sublattice. Given the higher symmetry of the metal ion distribution and the absence of any driver for distortion of the metal oxide tetrahedra, the existence of a second polymorph with $\mathrm{P}_{2}$ symmetry, but the same connectivity and metal distribution, seems unlikely. This suggests that the previous assignment of lower symmetry to the structure of $\mathrm{Li}_{2} \mathrm{MoO}_{4}$ may be erroneous. ${ }^{39}$

X-ray diffraction patterns collected from bulk samples of $\mathrm{Li}_{2} \mathrm{MoO}_{4}$ prepared by a variety of different methods can all be fitted using the model derived from the single crystal study. In addition to the high-temperature preparation and recrystallisation from aqueous solution, a highly crystalline sample of $\mathrm{Li}_{2} \mathrm{MoO}_{4}$ is produced by direct reaction between $\mathrm{LiOH} \cdot \mathrm{H}_{2} \mathrm{O}$ and $\mathrm{MoO}_{3}$. This reaction proceeds rapidly at room temperature: briefly mixing the reagents by grinding results in the rapid formation of highly crystalline product and complete conversion in less than 50 minutes to give several grams of $\mathrm{Li}_{2} \mathrm{MoO}_{4}$. Reaction between anhydrous lithium hydroxide and $\mathrm{MoO}_{3}$ proceeded much more slowly and is incomplete several weeks after thoroughly mixing and grinding the reagents together. This process was studied under 
ambient conditions and the slow rate of this reaction suggests that it proceeds by scavenging moisture from the air to form the hydrated material that can then undergo reaction with $\mathrm{MoO}_{3}$.

It is well established that the application of mechanical work to reagents can provide sufficient energy to allow diffusion and reaction in the absence of direct heating. ${ }^{20,41}$ The observation of highly crystalline product in sample B shows that lithium molybdate is readily formed at room temperature with the minimum input of mechanical energy necessary to ensure mixing of the finely ground reagents. This provides some evidence that the reaction is not activated by mechanical energy and this is even more strongly demonstrated by the calorimetric study of the progress of this reaction.

The formation of a tacky paste during the preparation of sample $\mathbf{A}$, and the slow rate of reaction of anhydrous lithium hydroxide, suggest that water plays a crucial role in this rapid room temperature reaction. In order to ensure the absence of liquid water and so prevent the reaction from taking place during mixing, a calorimetric study was performed after mixing the reagents at $-80{ }^{\circ} \mathrm{C}$. By preventing the immediate commencement of the reaction on contact between reagents in this manner, it was possible to mix the reagents without causing the reaction to begin. The temperature of the mixture increases steadily with heating and shows an exothermic event followed by an endothermic event occurring below room temperature. As the preparation of samples $\mathbf{A}$ and $\mathbf{B}$ show that $\mathrm{Li}_{2} \mathrm{MoO}_{4}$ is formed at room temperature and no other events occur below this temperature, it follows that the two processes that occur below this temperature represent reaction (1); the spontaneous formation of crystalline lithium molybdate from stable starting materials.

These data also provide an insight into the thermodynamic drivers behind this reaction. It could be imagined that the negative enthalpy change arising from the formation of an additional water molecule will provide a negative change in the free energy of the system and thus drive the formation of lithium molybdate. However, the data show that of the two 
transitions below room temperature it is the endotherm that dominates over the initial exotherm. We note that due to the incomplete reaction the energies of these transitions cannot be absolutely quantified, but the relative integrated signals show that the endothermic process absorbs more energy than the exothermic process produces.

As the reaction proceeds spontaneously it follows that the Gibbs Free Energy must be negative for the reaction. Clearly, this endothermic process must be more than compensated by the entropy change of the reaction. Consideration of the changes of state that occur show that this must be dominated by the formation of liquid water; the reagents are both crystalline solids whereas the majority of atoms in the products are found in liberated water. This change of state, and the introduction of the additional degrees of freedom associated with the liquid phase, will lead to a substantial increase in the entropy of the system and the calorimetry data suggest that it is this liberation of water from the crystal lattice of lithium hydroxide monohydrate that drives the reaction.

In addition to playing a role in the thermodynamics of the reaction, the water appears to be involved in the mechanism of the formation of lithium molybdate. As the particle morphology of samples $\mathbf{A}$ and $\mathbf{B}$ bear no resemblance to either of the two starting materials, the transformation of the reagents to a crystalline product must involve the destruction of the crystal lattices of both of the starting materials. Therefore although this reaction occurs between two solids to give a solid product it should not be considered a solid state reaction. In this respect it is similar to a number of reactions between two organic solids that proceed via the formation of liquid phase. ${ }^{42}$

As both reagents are stable under ambient conditions it is clear that contact between the two reagents initiates the reaction. Molybdenum trioxide is insoluble in water, but readily dissolves in alkali solution. ${ }^{43}$ This suggests that if a few layers of $\mathrm{LiOH} \cdot \mathrm{H}_{2} \mathrm{O}$ at the interface between the reagents dissolve, either in water of crystallisation or atmospheric moisture, this 
localised solution will be sufficiently basic to dissolve the surface layers of $\mathrm{MoO}_{3}$ to give a local, highly concentrated solution of $\mathrm{Li}^{+}$and $\mathrm{MoO}_{4}{ }^{2-}$. Such a situation could lead to the formation of lithium molybdate at the interfacial regions between the two reagents by growing out of a miniscule, but highly concentrated, aqueous region between the two particles. The relatively dense structure of lithium molybdate and the highly constrained nature of the tetrahedral coordination environments of $\mathrm{Li}^{+}$and $\mathrm{Mo}^{6+}$ in this compound are likely to prohibit the facile transfer of cations through this phase. Consequently, the formation of this crystalline material between the two reagents could form a barrier to mass transport between the reagents and so impede the reaction.

The particles in $\mathbf{A}$ and $\mathbf{B}$ are more regular and smaller than either the particles obtained by recrystallisation or the monolithic material resulting from high temperature synthesis. Given the potential for product formation to block the passage of reagents this could be ascribed to limited mass transport. However, examination of the particles of A (formed rapidly by thoroughly mixing the reagents) and $\mathbf{B}$ (formed more slowly due to minimal grinding) shows that the particles are larger when the reaction proceeds more slowly, i.e. the particle growth is not self-terminated but instead is limited by kinetic considerations. In addition to giving a more regular particle size, sample A also shows significantly smaller particles than any of the other preparative routes.

The morphology and particle size of lithium molybdate have been manipulated previously by adjusting the concentrations and stirring rate whilst precipitating crystalline particles from ethanolic solution. ${ }^{16}$ By adjusting the concentrations of the solutions and the conditions of precipitation, rod shaped particles with a minimum size of $\sim 0.8 \times 4 \mu \mathrm{m}$ could be formed, i.e. considerably larger than those produced by grinding the reagents together. The direct reaction described here between the solid reagents gives a more rapid route to the ternary compound, without the use of solvents or heating and does not require any input of energy. It also yields 
a product with a small, regular particle size that is ideal for processing for either catalytic ${ }^{30}$ or mechanical applications. ${ }^{28}$ 


\section{Conclusion}

The direct reaction at room temperature between the two, stable, crystalline materials, lithium hydroxide monohydrate and molybdenum trioxide produces highly crystalline lithium molybdate within minutes. This facile reaction is driven by the entropy increase associated with the liberation of water from the crystalline lattice. The rapidity of the reaction severely limits particle growth leading to the production of relatively uniform particles in the $\mu \mathrm{m}$ dimensions. The particle morphology shows that this is not a topotactic reaction, but instead involves partial, localised dissolution of the reagents. Despite the unprecedented rapidity of this reaction, the key components of the thermodynamic driver (the water of crystallisation in the reagent(s) is absent in the product) and mechanisms (the reagents are soluble in water/alkali) are exceedingly common. This suggests that other ternary, or higher, materials may be easily accessible via direct reaction at room temperature between crystalline reagents.

\section{Acknowledgements}

The authors are grateful to the Royal Society for the provision of a University Research Fellowship to EJC and to the University of Strathclyde for funding. We would also like to thank Dr John Liggat for assistance with the calorimetric measurements, Dr Paul Edwards for collecting scanning electron micrographs, Mr James Morrow for assistance in collecting thermogravimetric data and ITI Energy (Scotland) for provision of the necessary equipment. 
Table 1 Experimental details of the crystallographic characterisation of a single crystal of $\mathrm{Li}_{2} \mathrm{MoO}_{4}$.

\section{Crystal data}

Chemical formula

Formula weight $\left(\mathrm{M}_{\mathrm{r}}\right)$

Cell setting

Space group

$T / \mathrm{K}$

$a / \AA$

$c / \AA$

$V / \AA^{3}$

$\mathrm{Z}$

$\mathrm{D}_{\mathrm{c}} / \mathrm{Mg} \mathrm{m}^{-3}$

Radiation type

$\mu \mathrm{mm}^{-1}$

Crystal form

Colour

Crystal size / $\mathrm{mm}$

Data collection

Data collection method Absorption correction

$T_{\min }$

$T_{\max }$

No. of (measured, independent, observed) reflections Criterion for observation

$R_{\text {int }}$

$\theta_{\max } /{ }^{\circ}$

\section{Refinement}

$R(F), w R\left(F^{2}\right), S$

No. of reflections

No. of parameters

Weighting scheme ${ }^{\mathrm{b}}$
$(\Delta / \sigma)_{\max }$
$\Delta \rho_{\operatorname{man}}, \Delta \rho_{\min } / \mathrm{e} \AA^{-3}$
${ }^{\mathrm{a}}$ based on symmetry-related measurements
${ }^{\mathrm{b}} \mathrm{P}=\left(\mathrm{F}_{\mathrm{o}}{ }^{2}+2 \mathrm{~F}_{\mathrm{c}}{ }^{2}\right) / 3$

$\mathrm{Li}_{2} \mathrm{MoO}_{4}$

173.82

Trigonal

$R \overline{3}$

$150(2)$

$14.3178(14)$

9.5757(9)

$1700.0(2)$

18

3.056

Mo-K $\alpha$

3.319

Columnar

Colourless

$0.30 \times 0.10 \times 0.10$

$\omega$

Multi-scan ${ }^{\mathrm{a}}$

0.536

0.718

$(3533,867,858)$

$I>2 \sigma(I)$

0.026

27.46

$0.016,0.036,1.259$

867

65

$w=1 /\left[\sigma^{2}\left(F_{\mathrm{o}}^{2}\right)+(0.0163 P)^{2}+2.0800 P\right]$

0.001

$0.39,-0.38$ 
Table 2 Structural parameters for $\mathrm{Li}_{2} \mathrm{MoO}_{4}$ derived from single crystal X-ray diffraction.

\begin{tabular}{cccccc}
\hline Atom & Site & $x$ & $y$ & $z$ & $U_{\mathrm{eq}} / \AA^{2}$ \\
\hline $\mathrm{Mo}$ & $18 f$ & $0.647338(13)$ & $0.118283(13)$ & $0.08391(2)$ & $0.00836(8)$ \\
$\mathrm{Li} 1$ & $18 f$ & $0.4550(3)$ & $0.1411(3)$ & $0.2472(4)$ & $0.0136(8)$ \\
$\mathrm{Li} 2$ & $18 f$ & $0.8560(3)$ & $0.3091(3)$ & $-0.0816(4)$ & $0.0138(8)$ \\
$\mathrm{O} 1$ & $18 f$ & $0.66476(11)$ & $0.00505(11)$ & $0.0847(2)$ & $0.0117(3)$ \\
$\mathrm{O} 2$ & $18 f$ & $0.57914(11)$ & $0.11951(11)$ & $0.2369(2)$ & $0.0122(3)$ \\
$\mathrm{O} 3$ & $18 f$ & $0.77690(12)$ & $0.23377(12)$ & $0.0835(2)$ & $0.0130(3)$ \\
$\mathrm{O} 4$ & $18 f$ & $0.57780(11)$ & $0.11881(11)$ & $-0.0686(2)$ & $0.0122(3)$ \\
\hline
\end{tabular}




\section{Figures Captions}

Figure 1 (i) A projection of the rhombohedral crystal structure of $\mathrm{Li}_{2} \mathrm{MoO}_{4}$ derived from analysis of single crystal diffraction data. The lithium and molybdenum, represented as white and black spheres respectively, reside in the centre of tetrahedra composed of oxide anions. These tetrahedral units are connected by corner-sharing via trigonally-coordinated oxide anions to 8 neighbouring tetrahedral units. $\mathrm{Li}_{2} \mathrm{MoO}_{4}$ has previously been reported to crystallise in the space groups $R \overline{3}$ (ii) and $\mathrm{P} 3_{2}$ (iii). The differences between these structures are illustrated in (ii) and (iii).

Figure 2 Observed (dots), calculated (top line) and difference (bottom line) X-ray powder diffraction patterns collected from (i) sample A and (ii) sample $\mathbf{C}$. The vertical marks indicate the allowed Bragg reflection positions. Sample A contains a small amount of $\mathrm{MoO}_{3}$ impurity. The most intense peak from this phase is indicated by an arrow and the reflections due to this phase are indicated by the upper set of tick marks.

Figure 3 Observed X-ray powder diffraction patterns collected from a 2:1 mixture of $\mathrm{LiOH} \cdot \mathrm{H}_{2} \mathrm{O}$ and $\mathrm{MoO}_{3}$ which were ground for 1 minute. The inset shows data collected over the course of ca. 40 mins: (i) after 10 mins, (ii) after 20 mins, (iii) after 30 mins and (iv) after 40 mins. The upper and lower tick marks indicate the allowed reflection positions for $\mathrm{MoO}_{3}$ and $\mathrm{Li}_{2} \mathrm{MoO}_{4}$, respectively. The most intense reflection due to $\mathrm{MoO}_{3}$ is indicated with an arrow. 
Figure 4 Calorimetric data collected on heating a 2:1 mixture of $\mathrm{LiOH} \cdot \mathrm{H}_{2} \mathrm{O}$ and $\mathrm{MoO}_{3}$ from $-80{ }^{\circ} \mathrm{C}$ to $200{ }^{\circ} \mathrm{C}$ at a rate of $1{ }^{\circ} \mathrm{C} \mathrm{min}^{-1}$. The reagents were introduced and mixed at $-80{ }^{\circ} \mathrm{C}$ and the experiment was conducted under a dynamic atmosphere of dry air. The heat flow is indicated by the continuous line and positive and negative values indicate exo- and endothermic processes respectively. The temperature is indicated by a broken line.

Figure 5 Scanning electron micrographs of $\mathrm{Li}_{2} \mathrm{MoO}_{4}$ recorded from (i) and (ii) sample A, (iii) and (iv) sample B, (v) and (vi) sample C, and (vii) and (viii) sample $\mathbf{D}$. 


\section{References}

1. C. N. R. Rao and J. Gopalakrishnan, New Directions in Solid State Chemistry, 2nd edn., Cambridge University Press, Cambridge, 1997.

2. $\quad$ E. J. Cussen and T. W. S. Yip, J. Solid State Chem., 2007, 180, 1832.

3. A. R. Armstrong and P. G. Bruce, Nature, 1996, 381, 499.

4. J.-F. Colin, V. Pralong, M. Hervieu, V. Caignaert and B. Raveau, J. Mater. Chem., $2008,18,3121$.

5. R. E. Schaak and T. E. Mallouk, Chem. Mater., 2002, 14, 1455.

6. S. Tahara, T. Ichikawa, G. Kajiwara and Y. Sugahara, Chem. Mater., 2007, 19, 2351.

7. L. Sebastian, R. S. Jayashree and J. Gopalakrishnan, J. Mater. Chem., 2003, 13, 1400.

8. D. J. D. Corcoran, D. P. Tunstall and J. T. S. Irvine, Solid State Ionics, 2000, 136-137, 297.

9. V. Caignaert, F. Millange, B. Domenges and B. Raveau, Chem. Mater., 1999, 11, 930.

10. M. A. Hayward, E. J. Cussen, J. B. Claridge, M. Bieringer, M. J. Rosseinsky, C. J. Kiely, S. J. Blundell, I. M. Marshall and F. L. Pratt, Science, 2002, 295, 1882.

11. Y. Tsujimoto, C. Tassel, N. Hayashi, T. Watanabe, H. Kageyama, K. Yoshimura, M. Takano, M. Ceretti, C. Ritter and W. Paulus, Nature, 2007, 450, 1062.

12. L. D. Aikens, R. K. Li and C. Greaves, Chem. Commun., 2000, 2129.

13. M. A. Hayward, Chem. Mater., 2005, 17, 670.

14. K. J. Lee and E. Iguchi, J. Solid State Chem., 1995, 114, 242.

15. C. N. Chervin, B. J. Clapsaddle, H. W. Chiu, A. E. Gash, J. H. Satcher and S. M. Kauzlarich, Chem. Mater., 2006, 18, 1928.

16. M. Inagachi, Y. Nishikawa and M. Sakai, J. Eur. Ceram. Soc., 1992, 10, 123.

17. V. Thangadurai, C. Knittlmayer and W. Weppner, Mater. Sci. Eng., B, 2004, 106, 228.

18. C. Peng, L. Gao, S. W. Yang and J. Sun, Chem. Commun., 2008, 5601.

19. W. T. Jeong and K. S. Lee, J. Power Sources, 2002, 104, 195.

20. F. Goutenoire, R. Retoux, E. Suard and P. Lacorre, J. Solid State Chem., 1999, 142, 228.

21. J. Wang, J. M. Xue, D. M. Wan and B. K. Gan, J. Solid State Chem., 2000, 154, 321.

22. I. P. Parkin and A. V. Komarov, Polyhedron, 1996, 15, 3117.

23. S. Perera, N. Zelenski and E. G. Gillan, Chem. Mater., 2006, 18, 2381.

24. I. P. Parkin, Transition Met. Chem., 2002, 27, 569. 
25. M. V. Kuznetsov, I. P. Parkin, D. J. Caruana and Y. G. Morozov, J. Mater. Chem., 2004, 14, 1377.

26. J. M. Córdoba, M. J. Sayagués, M. D. Alcalá and F. J. Gotor, J. Mater. Chem., 2007, 17,650 .

27. J. B. Wiley, E. G. Gillan and R. B. Kaner, Mater. Res. Bull., 1993, 28, 893.

28. A. Forsgren, Corrosion control through organic coatings, CRC Press, New York, 2006.

29. E. Cavalli, E. Zannoni, C. Mucchino, V. Carozzo, A. Toncelli and M. Tonelli, J. Opt. Soc. Am. B: Opt. Phys., 1999, 16, 1958.

30. A. Erdöhelyi, K. Fodor, R. Németh, A. Hancz and A. Oszkó, J. Catal., 2001, 199, 328.

31. L. Sebastian, Y. Piffard, A. K. Shukla, F. Taulelle and J. Gopalakrishnan, J. Mater. Chem., 2003, 13, 1797.

32. H. Nakajima, H. Watanabe and S. Fujitani, US Pat., 6346 348, 2002.

33. P. Tundo, P. Anastas, D. S. Black, J. Breen, T. Collins, S. Memoli, J. Miyamoto, M. Polyakoff and W. Tumas, Pure Appl. Chem., 2000, 72, 1207.

34. S. Sharma and R. N. P. Choudhary, J. Mater. Sci. Lett., 1999, 18, 669.

35. G. M. Sheldrick, Acta Crystallographica, 2008, A64, 112.

36. H. M. Rietveld, Acta Cryst., 1969, 2, 65.

37. A. C. Larson and R. B. von Dreele, General Structure Analysis System (GSAS), Los Alamos National Laboratories, 1990.

38. U. Kolitsch, Zeitschrift Fur Kristallographie, 2001, 216, 449.

39. A. V. Barinova, R. K. Rastsvetaeva, Y. V. Nekrasov and D. Y. Pushcharovskii, Dokl. Akad. Nauk, 2001, 376, 343.

40. W. H. Zachariasen, Norsk. Geol. Tidsskr., 1926, 9, 65.

41. L. J. Ning, Y. P. Wu, S. B. Fang, E. Rahm and R. Holze, J. Power Sources, 2004, 133, 229.

42. G. Rothenberg, A. P. Downie, C. L. Raston and J. L. Scott, J. Am. Ceram. Soc., 2001, 123, 8701 .

43. N. N. Greenwood and A. Earnshaw, Chemistry of the Elements, 2nd edn., Butterworth-Heinemann, Oxford, 1997. 


\section{Figures Captions}

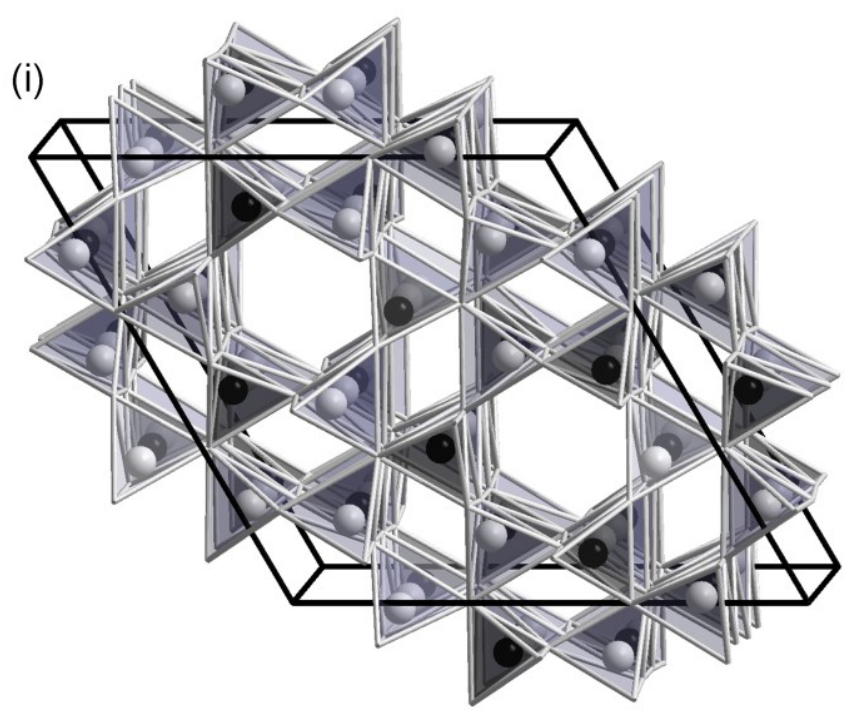

(ii)

$\mathrm{R} \overline{3}$

(iii)

$\mathrm{P}_{2}$
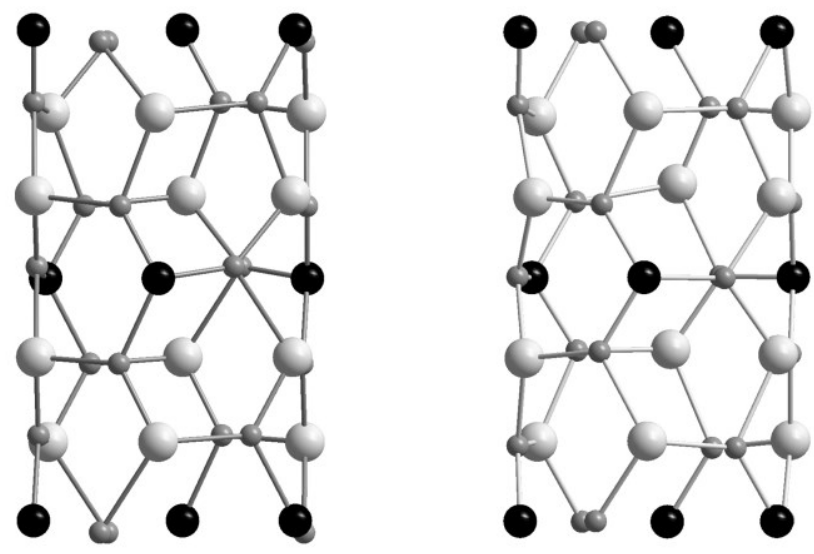

Figure 1 (i) A projection of the rhombohedral crystal structure of $\mathrm{Li}_{2} \mathrm{MoO}_{4}$ derived from analysis of single crystal diffraction data. The lithium and molybdenum, represented as white and black spheres respectively, reside in the centre of tetrahedra composed of oxide anions. These tetrahedral units are connected by corner-sharing via trigonally-coordinated oxide anions to 8 neighbouring tetrahedral units. $\mathrm{Li}_{2} \mathrm{MoO}_{4}$ has previously been reported to crystallise in the space groups $R \overline{3}$ (ii) and $\mathrm{P} 3_{2}$ (iii). The differences between these structures are illustrated in (ii) and (iii). 

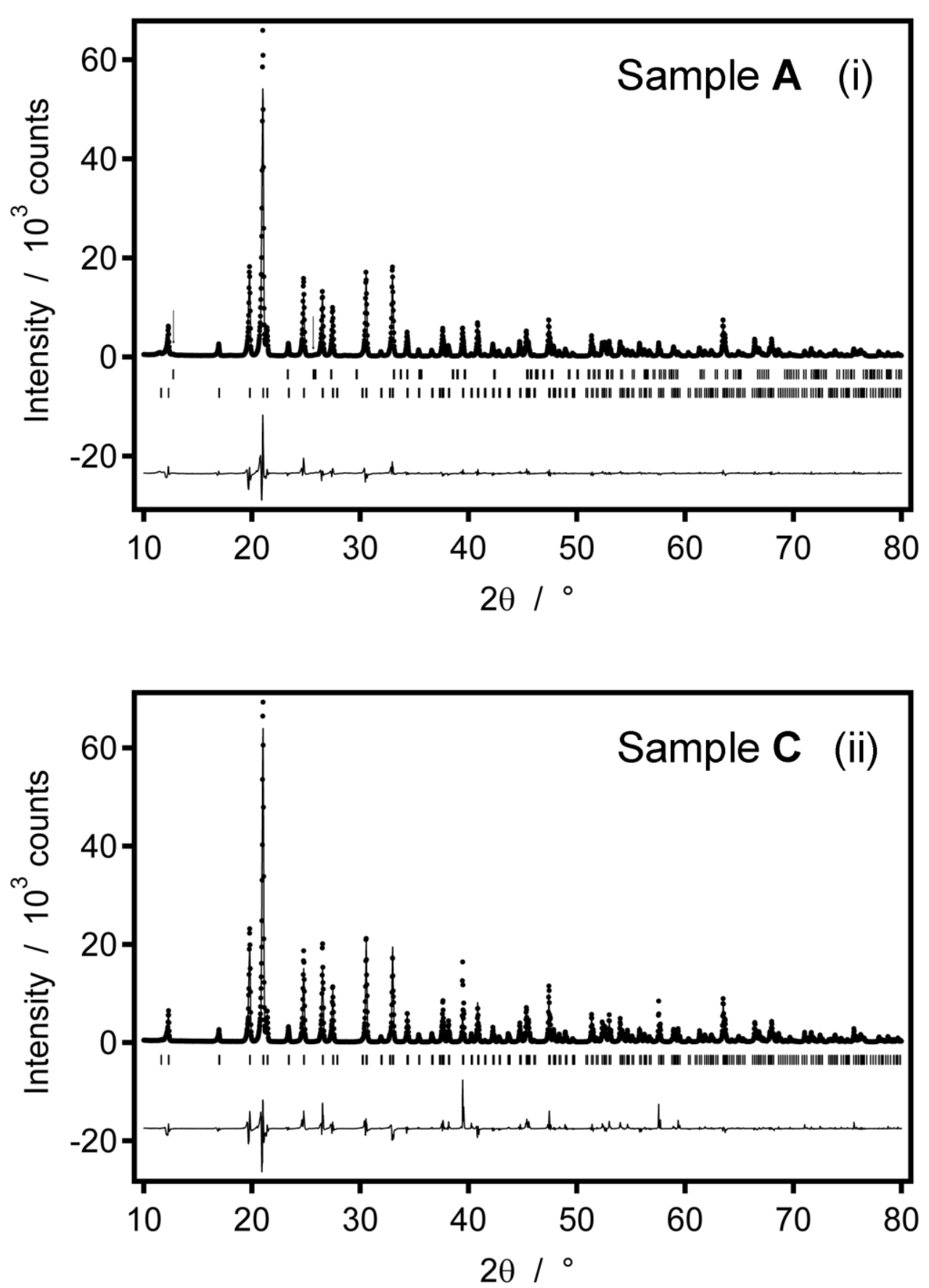

Figure 2 Observed (dots), calculated (top line) and difference (bottom line) X-ray powder diffraction patterns collected from (i) sample A and (ii) sample $\mathbf{C}$. The vertical marks indicate the allowed Bragg reflection positions. Sample A contains a small amount of $\mathrm{MoO}_{3}$ impurity. The most intense peak from this phase is indicated by an arrow and the reflections due to this phase are indicated by the upper set of tick marks. 


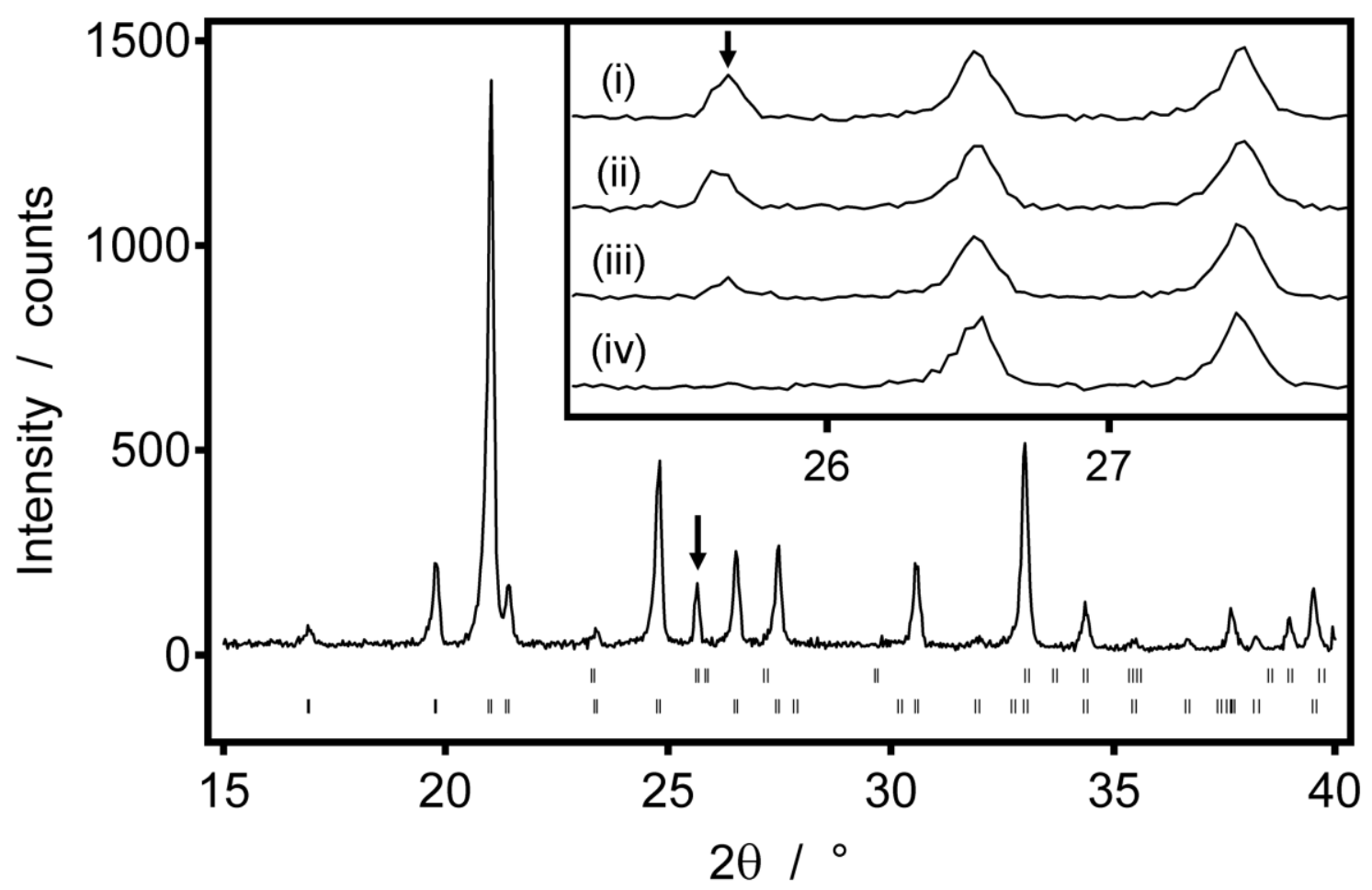

Figure 3 Observed X-ray powder diffraction patterns collected from a 2:1 mixture of $\mathrm{LiOH} \cdot \mathrm{H}_{2} \mathrm{O}$ and $\mathrm{MoO}_{3}$ which were ground for 1 minute. The inset shows data collected over the course of $c a .40$ mins: (i) after 10 mins, (ii) after 20 mins, (iii) after 30 mins and (iv) after 40 mins. The upper and lower tick marks indicate the allowed reflection positions for $\mathrm{MoO}_{3}$ and $\mathrm{Li}_{2} \mathrm{MoO}_{4}$, respectively. The most intense reflection due to $\mathrm{MoO}_{3}$ is indicated with an arrow. 


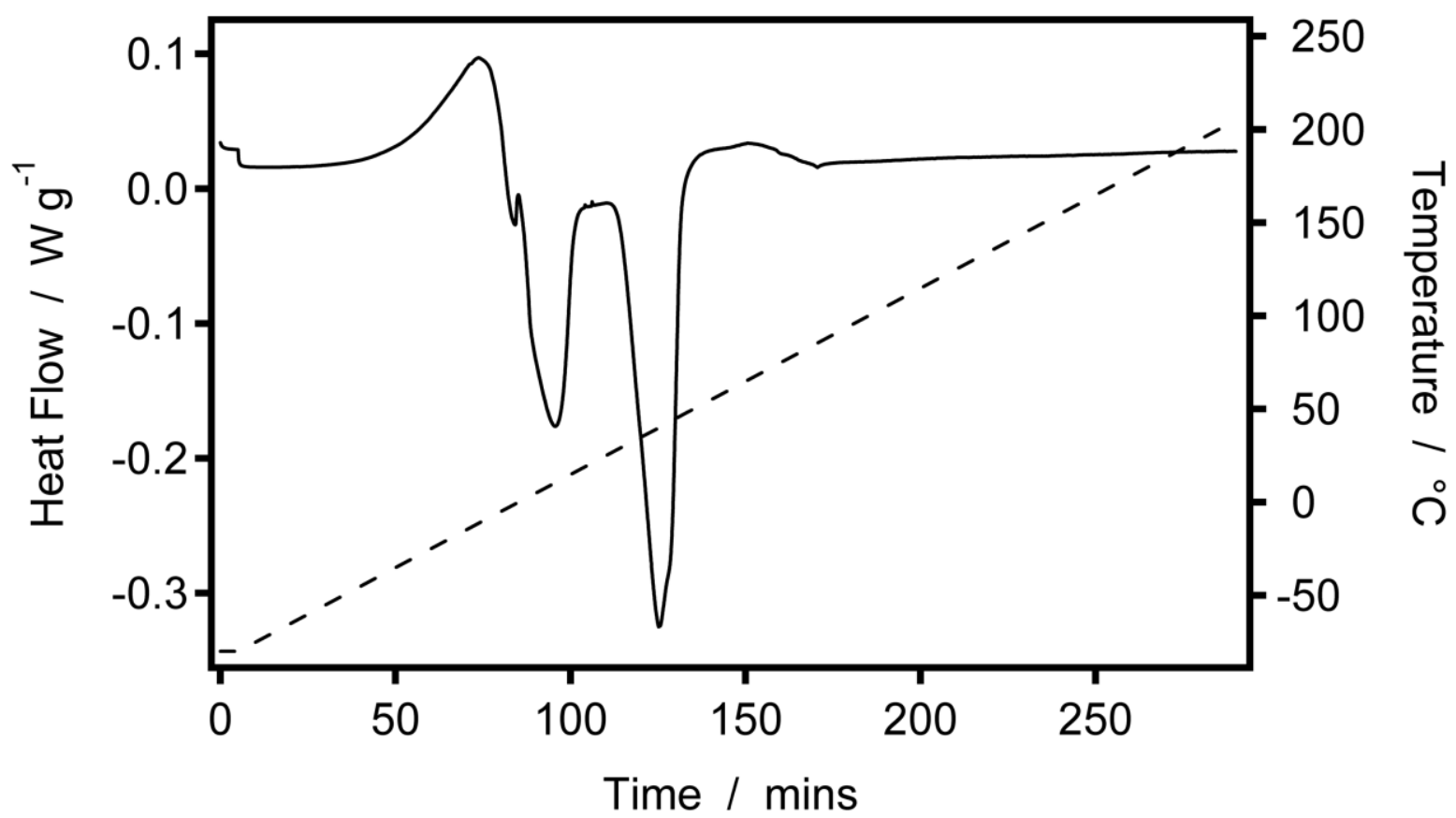

Figure 4 Calorimetric data collected on heating a 2:1 mixture of $\mathrm{LiOH} \cdot \mathrm{H}_{2} \mathrm{O}$ and $\mathrm{MoO}_{3}$ from $-80{ }^{\circ} \mathrm{C}$ to $200{ }^{\circ} \mathrm{C}$ at a rate of $1{ }^{\circ} \mathrm{C} \mathrm{min}^{-1}$. The reagents were introduced and mixed at $-80{ }^{\circ} \mathrm{C}$ and the experiment was conducted under a dynamic atmosphere of dry air. The heat flow is indicated by the continuous line and positive and negative values indicate exo- and endothermic processes respectively. The temperature is indicated by a broken line. 


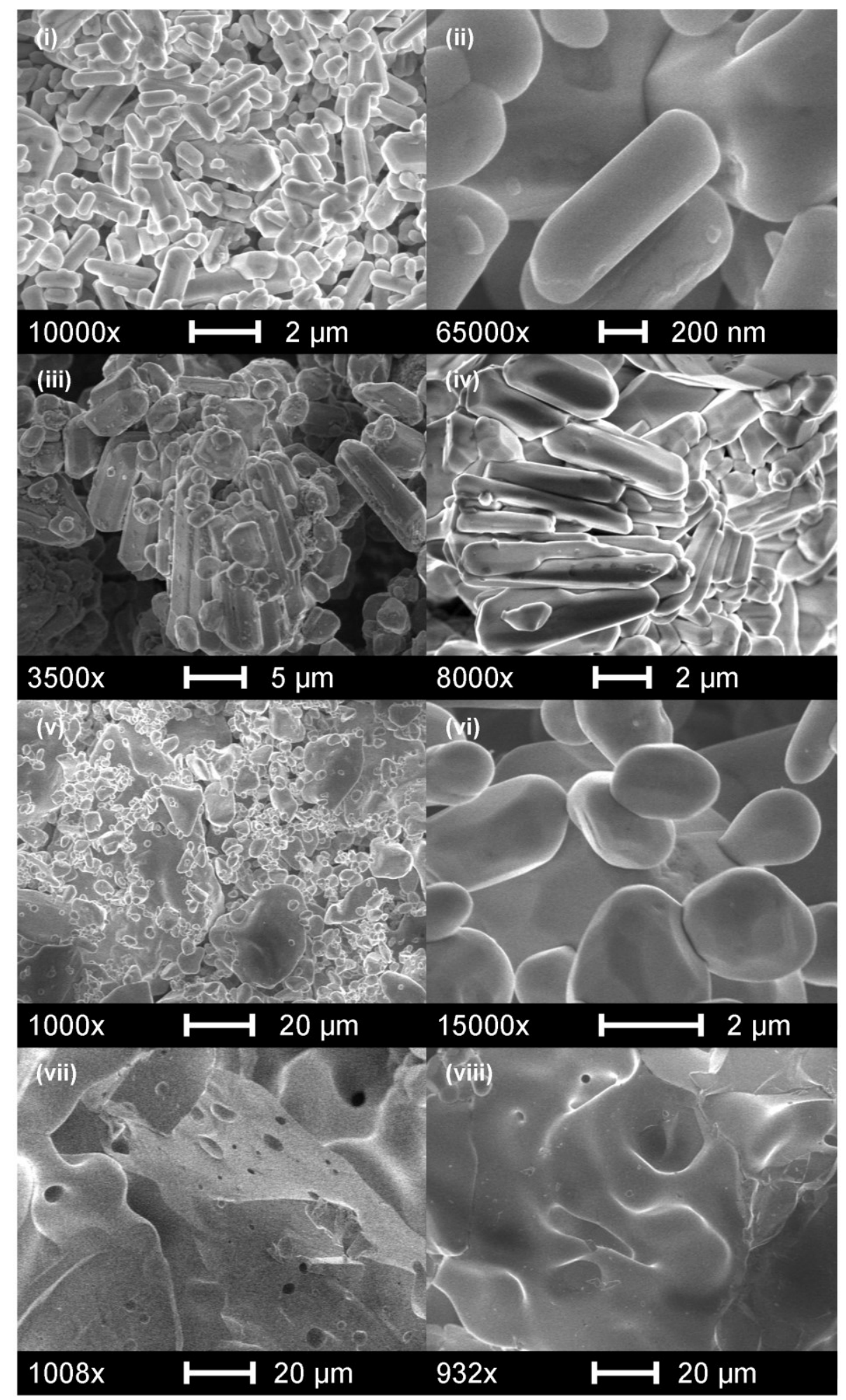

Figure 5 Scanning electron micrographs of $\mathrm{Li}_{2} \mathrm{MoO}_{4}$ recorded from (i) and (ii) sample $\mathbf{A}$,

(iii) and (iv) sample B, (v) and (vi) sample C, and (vii) and (viii) sample D. 